\title{
Screen-use, active-play and sleep behaviours in a nationally representative sample of infants, toddlers and pre-school children from the U.S.
}

\section{Abstract}

Introduction

Inadequate sleep, excessive screen-use and low-levels of physical activity may lead to an increased risk of childhood obesity. Caregivers during early childhood should limit screen-time, encourage active play and establish bedtime routines to ensure adequate sleep. The benefits of such may also extend to improved attention, learning, memory, psycho-social development and other factors. There is limited data on such lifestyle behaviours in infants and children in the U.S. Therefore, this analysis describes findings from the Feeding Infants and Toddlers Study (FITS) 2016.

\section{Methods}

FITS 2016 is a national, cross-sectional survey of caregivers of children aged 0-3.9 y in the U.S. Demographic data and information on feeding practices, sleep, physical activity and screen-use were collected using a questionnaire $(n=4380)$. Differences in behaviours across age groups were assessed using a one-way ANOVA or chi-squared tests.

\section{Results}

Children older than 12 months slept for an average of $9.6(\mathrm{sd}=2.0)$ hours per night, which was significantly higher than infants $(0$ $11.9 \mathrm{mo})$ who slept for $8.9(\mathrm{sd}=2.1)$ hours per night $(\mathrm{P}<0.001)$. All children slept for approximately one hour during the day. There were no significant difference in the weekly hours spent in active play (mean $=20.8, \mathrm{sd}=16.5$ ) between younger and older children, while participation in sports was higher among older versus younger children. However, time spent playing video games, watching TV videos/DVDs and using a screen was significantly higher in older versus younger children $(\mathrm{P}<0.001)$. For example, infants spent 7.8 hours per week watching TV, compared to 9.4 hours in 3 year old children $(\mathrm{P}<0.001)$. Approximately $65 \%$ of caregivers reported that they did not allow electronics in the bedroom, however this decreased with increasing age $(\mathrm{P}=0.036)$. Consequently, $5 \%$ of infants $(0-11.9 \mathrm{mo})$ viewed a tablet or smartphone in their bedroom, compared to $26 \%$ of 3 year old children $(\mathrm{P}<0.001)$.

\section{Discussion}

Recent guidelines from the American Academy for Pediatrics (AAP) recommend that children younger than 18 months avoid use of screen media, and that children aged 2-5 should limit screen-time to one hour per day. This data from the US FITS suggests that children are exceeding these recommendations. However, the current population are meeting the AAP physical activity recommendation of minimum 60 minutes/day. Furthermore, infants and children in this population are also failing to meet the AAP sleep recommendations. Therefore, further emphasis needs to be placed on the importance of limiting screen-time and promoting physical activity and positive sleep behaviours in this young population.

\section{Conflict of Interest}

EFJ, YL and BK are Nestlé employees. 